\title{
An Explicit MOT Scheme for Solving the TD-EFVIE on Nonlinear and Dispersive Scatterers
}

\author{
Sadeed B. Sayed ${ }^{1}$, H. Arda Ulku ${ }^{2}$, and Hakan Bagci ${ }^{1}$ \\ ${ }^{1}$ Division of Computer, Electrical and Mathematical Science and Engineering (CEMSE) \\ King Abdullah University of Science and Technology (KAUST), Thuwal, 23955-6900, Saudi Arabia \\ e-mails: \{sadeed.sayed, hakan.bagci\} @ kaust.edu.sa \\ ${ }^{2}$ Department of Electronics Engineering, Gebze Technical University, Gebze, Kocaeli, Turkey \\ e-mail: haulku@gtu.edu.tr
}

\begin{abstract}
An explicit marching-on-in-time (MOT) scheme for solving the time domain electric field volume integral equation (TD-EFVIE) on nonlinear and dispersive scatterers is described. The unknown electric field intensity, electric flux density, and polarization densities representing Kerr nonlinearity along with Lorentz dispersion relation, all of which are induced inside the scatterer upon excitation, are expanded using half and full Schaubert-Wilton-Glisson functions in space. The TD-EFVIE and the constitutive relations between polarization, field, and flux terms are cast in the form of a first-order ordinary differential equation. The resulting matrix system is integrated in time using a predictor-corrector scheme to obtain the time dependent unknown expansion coefficients. The resulting MOT scheme is explicit and accounts for nonlinearity by simple function evaluations.
\end{abstract}

\section{INTRODUCTION}

Numerical schemes, which are developed for computing electromagnetic fields on nonlinear and dispersive scatterers, represent the constitutive relation between field intensity, and flux and polarization density, which are induced inside the scatterer upon excitation, in the form of a nonlinear secondorder ordinary differential equation (NL-S-ODE). Then, the NL-S-ODE is coupled to the Maxwell equations and the resulting system is solved for the field intensity, and flux and polarization density. For example, in [1], a finite difference time domain (FDTD) scheme for solving the coupled system of the Maxwell curl equations and the NL-S-ODE has been developed. Even though, time-domain volume integral equation (TDVIE) solvers offer several advantages over FDTD schemes, their formulation and implementation for nonlinear dispersive scatterers have not been reported so far because the implicit marching-on-in-time (MOT) schemes used for TDVIEs can not be easily modified to account for nonlinearities in the constitutive relation.

In this work, the coupled system of time domain electric field volume integral equation (TD-EFVIE) and an NL-S-ODE accounting for Kerr nonlinearity along with Lorentz dispersion relation is solved using an explicit MOT scheme [2]. The proposed solver expands the unknown electric field intensity and flux density and polarization densities using half and full Schaubert-Wilton-Glisson (SWG) basis functions in space [3]. The TD-EFVIE and the constitutive relations between polarization, field, and flux terms are cast in the form of a first- order ODE which is integrated in time using a linear multistep, $P E(C E)^{m}$ scheme, to yield the unknown time dependent expansion coefficients. The explicitness of the resulting MOT scheme allows for easy incorporation of the nonlinearity as a simple function evaluation on the right-hand side without the need for a Newton-like nonlinear solver.

\section{Formulation}

Let $V$ denote the volume of a nonlinear dispersive scatterer residing in an unbounded background medium with permeability $\mu_{0}$ and permittivity $\varepsilon_{0}$. The scatterer is assumed to be non-magnetic and exhibit Kerr nonlinearity along with Lorentz dispersion. It is excited by a band-limited incident electric field $\mathbf{E}^{\text {inc }}(\mathbf{r}, t)$. Upon excitation, electric field intensity $\mathbf{E}(\mathbf{r}, t)$ and electric flux density $\mathbf{D}(\mathbf{r}, t)$ are induced in $V . \mathbf{E}(\mathbf{r}, t)$ and $\mathbf{D}(\mathbf{r}, t)$ satisfy the TD-EFVIE [3]:

$$
\partial_{t} \mathbf{E}^{\text {inc }}(\mathbf{r}, t)=\partial_{t} \mathbf{E}(\mathbf{r}, t)-\mathcal{L}\{\mathbf{E}(\mathbf{r}, t)\}+\varepsilon_{0}^{-1} \mathcal{L}\{\mathbf{D}(\mathbf{r}, t)\}
$$

Here, the volume integral operator $\mathcal{L}\{\cdot\}$ is given by

$$
\mathcal{L}\{\mathbf{X}(\mathbf{r}, t)\}=\mu_{0} \varepsilon_{0} \int_{V} \frac{\partial_{t}^{3} \mathbf{X}\left(\mathbf{r}^{\prime}, \tau\right)}{4 \pi R} d s^{\prime}-\nabla \int_{V} \frac{\nabla^{\prime} \cdot \partial_{t} \mathbf{X}\left(\mathbf{r}^{\prime}, \tau\right)}{4 \pi R} d s^{\prime}
$$

where $\tau=t-R / c_{0}$ is the retarded time, $R=\left|\mathbf{r}-\mathbf{r}^{\prime}\right|$ is the distance between observer and source points $\mathbf{r}$ and $\mathbf{r}^{\prime}$ and $c_{0}$ is the speed of light in the background medium. $\mathbf{D}(\mathbf{r}, t)$ and $\mathbf{E}(\mathbf{r}, t)$ are related by the constitutive relation

$$
\mathbf{D}(\mathbf{r}, t)=\varepsilon_{0} \varepsilon_{\infty} \mathbf{E}(\mathbf{r}, t)+\mathbf{P}^{\mathrm{L}}(\mathbf{r}, t)+\varepsilon_{0} \chi|\mathbf{E}(\mathbf{r}, t)|^{2} \mathbf{E}(\mathbf{r}, t) .
$$

Here, $\varepsilon_{\infty}$ is the 'infinite frequency' dielectric constant of the scatterer and $\chi$ is the third-order Kerr coefficient. The Lorentz polarization density $\mathbf{P}^{\mathrm{L}}(\mathbf{r}, t)$ satisfies the first-order ODEs:

$\partial_{t} \mathbf{P}^{\mathrm{L}}(\mathbf{r}, t)=\mathbf{P}^{\hat{\mathrm{L}}}(\mathbf{r}, t)$

$\partial_{t} \mathbf{P}^{\hat{\mathrm{L}}}(\mathbf{r}, t)=-\omega_{\mathrm{p}}^{2} \mathbf{P}^{\mathrm{L}}(\mathbf{r}, t)-2 \delta_{\mathrm{p}} \mathbf{P}^{\hat{\mathrm{L}}}(\mathbf{r}, t)+\varepsilon_{0} \Delta \varepsilon_{\mathrm{p}} \omega_{\mathrm{p}}^{2} \mathbf{E}(\mathbf{r}, t)$.

The auxiliary variable $\mathbf{P}^{\hat{\mathrm{L}}}(\mathbf{r}, t)$ is introduced to reduce the second-order ODE, which originally relate $\mathbf{E}(\mathbf{r}, t)$ to $\mathbf{P}^{\mathrm{L}}(\mathbf{r}, t)$, to the above first-order ODEs [1]. In [3], $\omega_{\mathrm{p}}, \tau_{\mathrm{p}}, \delta_{\mathrm{p}}, \Delta \varepsilon_{\mathrm{p}}$, are the pole frequency, the pole relaxation time, the damping coefficient, and the change in permittivity, respectively. 
To solve the coupled equations (1)-(3), first $V$ is discretized into tetrahedrons and unknowns are expanded as

$$
\begin{aligned}
\mathbf{D}(\mathbf{r}, t) & =\sum_{n=1}^{N_{\mathrm{f}}}\{\bar{D}(t)\}_{n} \mathbf{f}_{n}^{\mathrm{f}}(\mathbf{r}) \\
\mathbf{E}(\mathbf{r}, t) & =\sum_{n=1}^{N_{\mathrm{h}}}\{\bar{E}(t)\}_{n} \mathbf{f}_{n}^{\mathrm{h}}(\mathbf{r}) \\
\partial_{t} \mathbf{E}(\mathbf{r}, t) & =\sum_{n=1}^{N_{\mathrm{h}}}\left\{\bar{E}^{\partial}(t)\right\}_{n} \mathbf{f}_{n}^{\mathrm{h}}(\mathbf{r}) \\
\mathbf{P}^{\mathrm{q}}(\mathbf{r}, t) & =\sum_{n=1}^{N_{\mathrm{h}}}\left\{\bar{P}^{\mathrm{q}}(t)\right\}_{n} \mathbf{f}_{n}^{\mathrm{h}}(\mathbf{r}) \\
\partial_{t} \mathbf{P}^{\mathrm{q}}(\mathbf{r}, t) & =\sum_{n=1}^{N_{\mathrm{h}}}\left\{\bar{P}^{\mathrm{q} \partial}(t)\right\}_{n} \mathbf{f}_{n}^{\mathrm{h}}(\mathbf{r})
\end{aligned}
$$

where $\mathrm{q} \in\{\mathrm{L}, \hat{\mathrm{L}}\}, \mathbf{f}_{n}^{\mathrm{f}}(\mathbf{r})$ and $\mathbf{f}_{n}^{\mathrm{h}}(\mathbf{r})$ are the full and half SWG functions [3], and $\bar{D}(t), \bar{E}(t)$, and $\bar{P}^{\mathrm{q}}(t)$ are the unknown time dependent coefficients. Inserting (4a)-(4c) into (1) and testing the resulting equation by $\mathbf{f}_{n}^{\mathrm{h}}(\mathbf{r}), m=1, . ., N^{\mathrm{h}}$ at discrete times $j \Delta t$ (with $\Delta t$ being the time step) yield:

$$
\overline{\bar{G}}^{\mathrm{hh}} \bar{E}_{j}^{\partial}=\bar{V}_{j}^{\mathrm{inc}}+\sum_{i=0}^{j} \overline{\bar{Z}}_{j-i}^{\mathrm{hh}} \bar{E}_{i}-\varepsilon_{0}^{-1} \sum_{i=0}^{j} \overline{\bar{Z}}_{j-i}^{\mathrm{hf}} \bar{D}_{i}
$$

where $\bar{D}_{j}=\bar{D}(j \Delta t), \bar{E}_{j}=\bar{E}(j \Delta t)$, and $\bar{E}_{j}^{\partial}=\bar{E}_{j}^{\partial}(j \Delta t)$ and the entries of $\overline{\bar{Z}}_{j-i}^{\text {uv }}, \overline{\bar{G}}^{\text {uv }}, \mathrm{u}, \mathrm{v} \in\{\mathrm{h}, \mathrm{f}\}$, and $\bar{V}_{j}^{\text {inc }}$ are given by

$$
\begin{aligned}
& \left\{\overline{\bar{Z}}_{j-i}^{\mathrm{uv}}\right\}_{m, n}=\left.\int_{V_{m}} \mathbf{f}_{m}^{\mathrm{u}}(\mathbf{r}) \cdot \mathcal{L}\left\{\mathbf{f}_{n}^{\mathrm{v}}(\mathbf{r}) T_{i}(t)\right\}\right|_{t=j \Delta t} d s \\
& \left\{\overline{\bar{G}}^{\mathrm{uv}}\right\}_{m, n}=\int_{V_{m}} \mathbf{f}_{m}^{\mathrm{u}}(\mathbf{r}) \cdot \mathbf{f}_{n}^{\mathrm{v}}(\mathbf{r}) d s \\
& \left\{\bar{V}_{j}^{\mathrm{inc}}\right\}_{m}=\left.\int_{V_{m}} \mathbf{f}_{m}^{\mathrm{h}}(\mathbf{r}) \cdot \partial_{t} \mathbf{E}^{\mathrm{inc}}(\mathbf{r}, t)\right|_{t=j \Delta t} d s .
\end{aligned}
$$

Here, $V_{m}$ is the support of $\mathbf{f}_{m}^{\mathrm{u}}(\mathbf{r}), T_{i}(t)=T(t-i \Delta t)$, where $T(t)$ is the approximate prolate spherical wave function [4], used for interpolating from $\bar{D}_{i}$ and $\bar{E}_{i}$ in order to accurately account for the time retardation in $\mathcal{L}\{\cdot\}$. Inserting $4 \mathrm{4a}$, , 4b , and (4d) into (2), and testing the resulting equation with $\mathbf{f}_{n}^{\mathbf{f}}(\mathbf{r})$, $m=1, . ., N^{\mathrm{f}}$ at discrete times $j \Delta t$ yield:

$$
\overline{\bar{G}}^{\mathrm{ff}} \bar{D}_{j}=\overline{\bar{G}}^{\mathrm{fh}}\left(\varepsilon_{0} \varepsilon_{\infty} \bar{E}_{j}+\bar{P}_{j}^{\mathrm{L}}+\alpha \varepsilon_{0} \chi\left|\bar{E}_{j}\right|^{2} \bar{E}_{j}\right)
$$

where $\bar{P}_{j}^{\mathrm{q}}=\bar{P}^{\mathrm{q}}(j \Delta t)$. Inserting (4b)-4e into (3) and testing the resulting equations at discrete times $j \Delta t$ yield:

$$
\begin{aligned}
& \bar{P}_{j}^{\mathrm{L} \partial}=\bar{P}_{j}^{\hat{\mathrm{L}}} \\
& \bar{P}_{j}^{\hat{\mathrm{L}} \partial}=-\omega_{\mathrm{p}}^{2} \bar{P}_{j}^{\mathrm{L}}-2 \delta_{\mathrm{p}} \bar{P}_{j}^{\hat{\mathrm{L}}}+\varepsilon_{0} \Delta \varepsilon_{\mathrm{p}} \omega_{\mathrm{p}}^{2} \bar{E}_{j}
\end{aligned}
$$

where $\bar{P}_{j}^{\mathrm{q} \partial}=\bar{P}^{\mathrm{q} \partial}(j \Delta t)$. The first-order ODE system represented by (5)-(7), is integrated in time using a $P E(C E)^{m}$ scheme [2] to obtain unknowns $\bar{D}_{j}, \bar{E}_{j}$, and $\bar{P}_{j}^{\mathrm{q}}$.

\section{Numerical Results}

The proposed solver is used for analyzing scattering from a sphere and a cube. In both simulations, $\mathbf{E}^{\text {inc }}(\mathbf{r}, t)=\hat{\mathbf{x}} G(t-$ $\left.\mathbf{r} \cdot \hat{\mathbf{z}} / c_{0}\right)$, where $G(\mathbf{r}, t)=\cos \left[2 \pi f_{0}\left(t-t_{\mathrm{p}}\right)\right] e^{-\left(t-t_{\mathrm{p}}\right)^{2} /\left(2 \sigma^{2}\right)}$ is a Gaussian pulse with duration $\sigma=3 /\left(2 \pi f_{\mathrm{bw}}\right)$, center frequency $f_{0}$, bandwidth $f_{\mathrm{bw}}$, and delay $t_{\mathrm{p}}=8 \sigma$. In the first simulation, the scatterer is a unit sphere made of linear Lorentz material and centered at the origin, $\omega_{\mathrm{p}}=3 \times 10^{8} \mathrm{~s}^{-1}$, $\delta_{\mathrm{p}}=10^{6} \mathrm{~s}^{-1}, \varepsilon_{\infty}=2, \Delta \varepsilon_{\mathrm{p}}=3, f_{0}=150 \mathrm{MHz}, f_{\mathrm{bw}}=50$

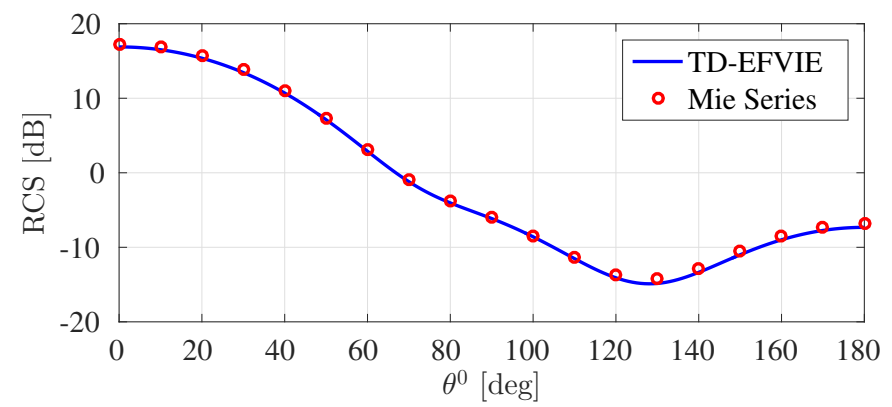

Fig. 1. RCS at $150 \mathrm{MHz}$ computed from the solutions obtained by the proposed solver and using the Mie series.

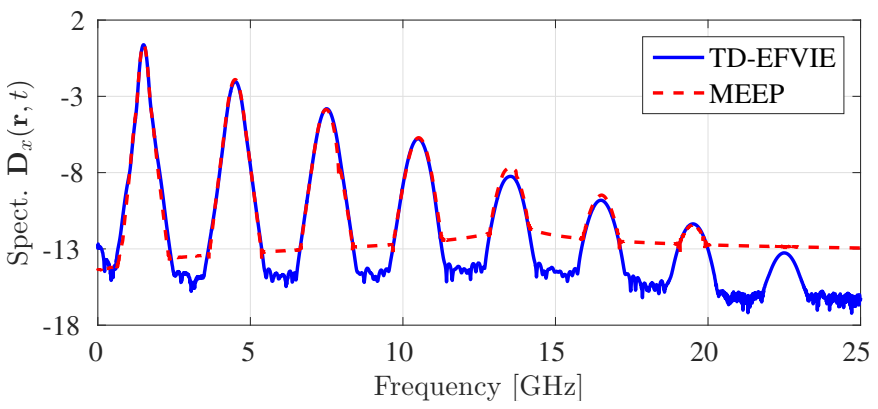

Fig. 2. Spectrum of $\mathbf{D}_{x}(\mathbf{r}, t)$ at $\mathbf{r}=(0,0,0) \mathrm{m}$ computed by the proposed solver and the FDTD-based MEEP.

$\mathrm{MHz}, N_{\mathrm{h}}=15028, N_{\mathrm{f}}=7752$, and $\Delta t=0.2$ ns. Fig. 1 shows that the radar cross section (RCS), which is computed at $150 \mathrm{MHz}$ by Fourier transforming the solution obtained by the proposed solver, matches well to that obtained from the Mie series solution.

In the second simulation, the scatterer is a nonlinear cube of dimension $0.1 \mathrm{~m}$ centered at the origin, $\varepsilon_{\infty}=2, \chi=0.01$, $f_{0}=1500 \mathrm{MHz}, f_{\mathrm{bw}}=150 \mathrm{MHz}, N_{\mathrm{h}}=7596, N_{\mathrm{f}}=4002$, and $\Delta t=13.3$ ps. Fig. 2 compares the spectrum of the $x$ - component of $\mathbf{D}(\mathbf{r}, t)$ at $\mathbf{r}=(0,0,0) \mathrm{m}$ computed by the proposed solver and the FDTD-based MEEP [5]. Results agree well.

\section{REFERENCES}

[1] P. M. Goorjian and A. Taflove, "Direct time integration of Maxwell's equations in nonlinear dispersive media for propagation and scattering of femtosecond electromagnetic solitons," Optics Letters, vol. 17, no. 3, p. 180, Feb. 1992.

[2] H. A. Ulku, H. Bagci, and E. Michielssen, "Marching on-in-time solution of the time domain magnetic field integral equation using a predictorcorrector scheme," IEEE Trans. Antennas Propag., vol. 61, pp. 41204131, Aug. 2013.

[3] G. Kobidze, J. Gao, B. Shanker, and E. Michielssen, "A fast time domain integral equation based scheme for analyzing scattering from dispersive objects," IEEE Trans. Antennas Propag., vol. 53, pp. 1215-1226, Mar. 2005.

[4] S. B. Sayed, H. A. Ulku, and H. Bagci, "A stable marching on-in-time scheme for solving the time domain electric field volume integral equation on high-contrast scatterers," IEEE Trans. Antennas Propag., vol. 63, pp. 3098-3110, July 2015.

[5] A. F. Oskooi, D. Roundy, M. Ibanescu, P. Bermel, J. D. Joannopoulos, and S. G. Johnson, "MEEP: A flexible free-software package for electromagnetic simulations by the FDTD method," Computer Physics Communications, vol. 181, no. 3, pp. 687-702, Mar. 2010. 